\title{
UNIQUENESS OF ENTIRE FUNCTIONS CONCERNING DIFFERENTIAL-DIFFERENCE POLYNOMIALS SHARING SMALL FUNCTIONS
}

\author{
GOUTAM HALDAR
}

\begin{abstract}
In this paper, we investigate the uniqueness problem of difference polynomials $f^{n}(z) P(f(z)) L_{c}(f)$ and $g^{n}(z) P(g(z)) L_{c}(g)$, where $L_{c}(f)=f(z+$ $c)+c_{0} f(z), P(z)$ is a polynomial with constant coefficients of degree $m$ sharing a small function with the notions of weakly weighted sharing and relaxed weighted sharing and obtained the corresponding results, which improve and extend some recent results due to Sahoo and Biswas (Tamkang J. Math., 49(2), 85-97 (2018)).
\end{abstract}

\section{Introduction}

Let $f$ and $g$ be two non-constant meromorphic functions defined in the open complex plane $\mathbb{C}$. If for some $a \in \mathbb{C} \cup\{\infty\}$, the zero of $f-a$ and $g-a$ have the same locations as well as same multiplicities, we say that $f$ and $g$ share the value $a$ $\mathrm{CM}$ (counting multiplicities). If we do not consider the multiplicities, then $f$ and $g$ are said to share the value $a$ IM (ignoring multiplicities). We adopt the standard notations of the Nevanlinna theory of meromorphic functions (see [5, 10, 20]). For a non-constant meromorphic function $f$, we denote by $T(r, f)$ the Nevanlinna characteristic function of $f$ and by $S(r, f)$ any quantity satisfying $S(r, f)=o\{T(r, f)\}$ as $r \rightarrow \infty$ outside of an exceptional set of finite linear measure. We say that $\alpha(z)$ is a small function of $f$, if $\alpha(z)$ is a meromorphic function satisfying $T(r, \alpha(z))=S(r, f)$. We denote by $E_{k)}(a, f)$ the set of all a-points of $f$ with multiplicities not exceeding $k$, where an a-point is counted according to its multiplicity. Also we denote by $\bar{E}_{k)}(a, f)$ the set of distinct a-points of $f$ with multiplicities not exceeding $k$. In addition, we need the following definitions.

Definition 1.1. [6] Let $p$ be a positive integer and $a \in \mathbb{C} \cup\{\infty\}$.

(i) $N(r, a ; f \mid \geq p) \overline{(N}(r, a ; f \mid \geq p))$ denotes the counting function (reduced counting function) of those a-points of $f$ whose multiplicities are not less than $p$.

(ii) $N(r, a ; f \mid \leq p) \overline{(N}(r, a ; f \mid \leq p))$ denotes the counting function (reduced counting function) of those a-points of $f$ whose multiplicities are not greater than $p$.

Definition 1.2. 7] Let $k$ be a positive integer or infinity. We denote by $N_{k}(r, a ; f)$ the counting function of a-points of $f$, where an a-point of multiplicity $m$ is counted

2010 Mathematics Subject Classification. 30D35.

Key words and phrases. Enitire function, difference operator, small function, weakly weighted sharing, relaxed weighted sharing. 
$m$ times if $m \leq k$ and $k$ times if $m>k$. Then

$$
N_{k}(r, a ; f)=\bar{N}(r, a ; f)+\bar{N}(r, a ; f \mid \geq 2)+\ldots+\bar{N}(r, a ; f \mid \geq k) .
$$

Clearly, $N_{1}(r, a ; f)=\bar{N}(r, a ; f)$.

Definition 1.3. Let $a \in \mathbb{C} \cup\{\infty\}$. We denote $N_{E}(r, a ; f, g)\left(N_{E}(r, a ; f, g)\right)$ by the counting function (reduced counting function) of all common zeros of $f-a$ and $g-a$ with the same multiplicities and by $N_{0}(r, a ; f, g)\left(N_{0}(r, a ; f, g)\right)$ the counting function (reduced counting function) of all common zeros of $f-a$ and $g-a$ ignoring multiplicities. If

$$
\bar{N}(r, a ; f)+\bar{N}(r, a ; g)-2 \bar{N}_{E}(r, a ; f, g)=S(r, f)+S(r, g),
$$

then we say that $f$ and $g$ share the value a "CM". If

$$
\bar{N}(r, a ; f)+\bar{N}(r, a ; g)-2 \bar{N}_{0}(r, a ; f, g)=S(r, f)+S(r, g),
$$

then we say that $f$ and $g$ share the value a "IM". Definition 4

Definition 1.4. 11] Let $f$ and $g$ share the value a "IM" and $k$ be a positive integer or infinity. Then $\bar{N}_{k)}^{E}(r, a ; f, g)$ denotes the reduced counting function of those apoints of $f$ whose multiplicities are equal to the corresponding a-points of $g$, and both of their multiplicities are not greater than $k . \bar{N}_{(k}^{0}(r, a ; f, g)$ denotes the reduced counting function of those a-points of $f$ which are a-points of $g$, and both of their multiplicities are not less than $k$.

Definition 1.5. 11] Let $a \in \mathbb{C} \cup\{\infty\}$ and $k$ be a positive integer or infinity. If

$$
\begin{gathered}
\bar{N}(r, a ; f \mid \leq k)-\bar{N}_{k)}^{E}(r, a ; f, g)=S(r, f), \\
\bar{N}(r, a ; g \mid \leq k)-\bar{N}_{k)}^{E}(r, a ; f, g)=S(r, g), \\
\bar{N}(r, a ; f \mid \geq k+1)-\bar{N}_{0}^{(k+1}(r, a ; f, g)=S(r, f), \\
\bar{N}(r, a ; g \mid \geq k+1)-\bar{N}_{0}^{(k+1}(r, a ; f, g)=S(r, g),
\end{gathered}
$$

or if $k=0$ and

$$
\begin{aligned}
& \bar{N}(r, a ; f)-\bar{N}_{0}(r, a ; f, g)=S(r, f), \\
& \bar{N}(r, a ; g)-\bar{N}_{0}(r, a ; f, g)=S(r, g),
\end{aligned}
$$

then we say that $f$ and $g$ share the value a weakly with weight $k$ and we write $f$ and $g$ share " $(a, k)$ ".

Definition 1.6. 1] Let $k$ be a positive integer and for $a \in \mathbb{C}-\{0\}, E_{k)}(a ; f)=$ $E_{k)}(a ; g)$. Let $z_{0}$ be a zero of $f(z)-a$ of multiplicity $p$ and a zero of $g(z)-a$ of multiplicity $q$. We denote by $\bar{N}_{L}(r, a ; f)\left(\bar{N}_{L}(r, a ; g)\right)$ the reduced counting function of those a-points of $f$ and $g$ for which $p>q \geq k+1(q>p \geq k+1)$, by $\bar{N}_{E}^{(k+1}(r, a ; f)$ the reduced counting function of those a-points of $f$ and $g$ for which $p=q \geq k+1$, by $\bar{N}_{f \geq k+1}(r, a ; f \mid g \neq a)$ the reduced counting functions of those a-points of $f$ and $g$ for which $p \geq k+1$ and $q=0$. 
Definition 1.7. [1] Let $k$ be a positive integer and fora $\in \mathbb{C}-\{0\}$, let $f, g$ share $a$ "IM". Let $z_{0}$ be a zero of $f(z)-a$ of multiplicity $p$ and a zero of $g(z)-a$ of multiplicity $q$. We denote by $\bar{N}_{f \geq k+1}(r, a ; f \mid g=m)$ the reduced counting functions of those a-points of $f$ and $g$ for which $p \geq k+1$ and $q=m$. We can define $\bar{N}_{L}(r, a ; f)\left(\bar{N}_{L}(r, a ; g)\right)$ and $\bar{N}_{E}^{(k+1}(r, a ; f)$ in a similar manner as defined in the previous definition.

Definition 1.8. [8] Let $a, b \in \mathbb{C} \cup\{\infty\}$. We denote by $N(r, a ; f \mid g=b)$ the counting function of those a-points of $f$, counted according to multiplicity, which are b-points of $g$.

Definition 1.9. 8 , Let $a, b \in \mathbb{C} \cup\{\infty\}$. We denote by $N(r, a ; f \mid g \neq b)$ the counting function of those a-points of $f$, counted according to multiplicity, which are not the b-points of $g$.

We define shift and difference operators of $f(z)$ by $f(z+c)$ and $\Delta_{c} f(z)=f(z+$ $c)-f(z)$, respectively. Note that $\Delta_{c}^{n} f(z)=\Delta_{c}^{n-1}\left(\Delta_{c} f(z)\right)$, where $c$ is a nonzero complex number and $n \geq 2$ is a positive integer. For further generalization of $\Delta_{c} f$, we now define the difference operator of an entire (meromorphic) function $f$ as $L_{c}(f)=f(z+c)+c_{0} f(z)$, where $c_{0}$ is a non-zero complex constant. Clearly, for the particular choice of the constant $c_{0}=-1$, we get $L_{c}(f)=\Delta_{c} f$.

In 2007, Banerjee and Mukherjee [1 introduced a new type of sharing known as relaxed weighted sharing which is weaker than weakly weighted sharing as follows.

Definition 1.10. [1] We denote by $N(r, a ; f|=p ; g|=q)$ the reduced counting function of common a-points of $f$ and $g$ with multiplicities $p$ and $q$, respectively.

Definition 1.11. [1] Let $a \in \mathbb{C} \cup\{\infty\}$ and $k$ be a positive integer or infinity. Suppose that $f$ and $g$ share the value a "IM". If for $p \neq q$,

$$
\sum_{p, q \leq k} N(r, a ; f|=p ; g|=q)=S(r),
$$

then we say that $f$ and $g$ share the value a with weight $k$ in a relaxed manner and we write $f$ and $g$ share $(a, k)^{*}$

Let $P(z)=a_{m} z^{m}+a_{m-1} z^{m-1}+\ldots+a_{0}$ be a nonzero polynomial of degree $m$, where $a_{m}(\neq 0), a_{m-1}, \ldots, a_{0}(\neq 0)$ are complex constants and $m$ is a positive integer.

In 1959, Hayman [4] proved the following result.

Theorem A. 4 Let $f$ be a transcendental entire function and let $n(\geq 1)$ be an integer. Then $f^{n} f^{\prime}=1$ has infinitely many solutions.

Regarding uniqueness of the above theorem, Yang and Hua [19], in 1997 obtained the following result.

Theorem B. 19] Let $f$ and $g$ be two non-constant entire functions, $n \geq 6$ a positive integer. If $f^{n} f^{\prime}$ and $g^{n} g^{\prime}$ share $1 C M$, then either $f(z)=c_{1} e^{c z}, g(z)=c_{2} e^{-c z}$, where $c_{1}, c_{2}$ and $c$ are three constants satisfying $\left(c_{1} c_{2}\right)^{n+1} c^{2}=-1$ or $f \equiv \operatorname{tg}$ for a constant $t$ satisfying $t^{n+1}=1$.

In 2002, Fang and Fang [3] extends Theorem B in the following manner

Theorem C. 3] Let $f$ and $g$ be two non-constant entire functions, and let $n(\geq 8)$ be an integer. If $f^{n}(f-1) f^{\prime}$ and $g^{n}(g-1) g^{\prime}$ share $1 C M$, then $f \equiv g$. 
In 2004, Lin and Yi [1] extended Theorem $\mathrm{C}$ by replacing value sharing with fixed point sharing and obtained the following result.

Theorem D. [11] Let $f$ and $g$ be two transcendental entire functions, and let $n(\geq 7)$ be an integer. If $f^{n}(f-1) f^{\prime}$ and $g^{n}(g-1) g^{\prime}$ share $z C M$, then $f \equiv g$.

In 2010, Zhang [21] obtained the following result by replacing $f^{\prime}(z)$ with $f(z+c)$, where $c$ is a non-zero constant.

Theorem E. 21] Let $f$ and $g$ be two transcendental entire functions of finite order, and $\alpha(z)(\not \equiv 0, \infty)$ be a small function with respect to both $f$ and $g$. Suppose that $c$ is a nonzero complex constant and $n \geq 7$ be an integer. If $f^{n}(z)(f(z)-1) f(z+c)$ and $g^{n}(z)(g(z)-1) g(z+c)$ share $\alpha(z) C M$, then $f(z) \equiv g(z)$.

Recently, Meng 13 improved Theorem E by relaxing the nature of sharing the small function and obtained following results.

Theorem F. 13 Let $f$ and $g$ be two transcendental entire functions of finite order, and $\alpha(z)(\not \equiv 0, \infty)$ be a small function with respect to both $f$ and $g$. Suppose that $c$ is a nonzero complex constant and $n \geq 7$ is an integer. If $f^{n}(z)(f(z)-1) f(z+c)$ and $g^{n}(z)(g(z)-1) g(z+c)$ share " $(\alpha(z), 2)$ ", then $f(z) \equiv g(z)$.

Theorem G. 13 Let $f$ and $g$ be two transcendental entire functions of finite order, and $\alpha(z)(\not \equiv 0, \infty)$ be a small function with respect to both $f$ and $g$. Suppose that $c$ is a nonzero complex constant and $n \geq 10$ is an integer. If $f^{n}(z)(f(z)-1) f(z+c)$ and $g^{n}(z)(g(z)-1) g(z+c)$ share $(\alpha(z), 2)^{*}$, then $f(z) \equiv g(z)$.

Theorem H. 13] Let $f(z)$ and $g(z)$ be two transcendental entire functions of finite order, and $\alpha(z)(\not \equiv 0, \infty)$ be a small function with respect to both $f(z)$ and $g(z)$. Suppose that $c$ is a nonzero complex constant and $n \geq 16$ is an integer. If $\bar{E}_{2)}\left(\alpha(z), f^{n}(z)(f(z)-1) f(z+c)\right)=\bar{E}_{2)}\left(\alpha(z), g^{n}(z)(g(z)-1) g(z+c)\right)$, then $f(z) \equiv g(z)$.

In 2018, Sahoo and Biswas [16] further extended Theorems $\mathrm{E}-\mathrm{H}$ in the following.

Theorem I. [16] Let $f(z)$ and $g(z)$ be two transcendental entire functions of finite order, and $\alpha(z)(\not \equiv 0, \infty)$ be a small function with respect to both $f(z)$ and $g(z)$ with finitely many zeros. Suppose that $c$ is a non-zero complex constant, $n, k(\geq 0)$ and $m(\geq 1)$ are integers such that $n \geq 2 k+m+6$. If $\left(f^{n}(z)\left(f^{m}(z)-1\right) f(z+c)\right)^{(k)}$ and $\left(g^{n}(z)\left(g^{m}(z)-1\right) g(z+c)\right)^{(k)}$ share " $(\alpha(z), 2)$ ", then $f(z) \equiv \operatorname{tg}(z)$, where $t^{m}=1$.

Theorem J. [16] Let $f(z)$ and $g(z)$ be two transcendental entire functions of finite order, and $\alpha(z)(\not \equiv 0, \infty)$ be a small function with respect to both $f(z)$ and $g(z)$ with finitely many zeros. Suppose that $c$ is a non-zero complex constant, $n, k(\geq 0)$ and $m(\geq 1)$ are integers such that $n \geq 3 k+2 m+8$. If $\left(f^{n}(z)\left(f^{m}(z)-1\right) f(z+c)\right)^{(k)}$ and $\left(g^{n}(z)\left(g^{m}(z)-1\right) g(z+c)\right)^{(k)}$ share $(\alpha(z), 2)^{*}$, then $f(z) \equiv \operatorname{tg}(z)$, where $t^{m}=1$.

Theorem K. [16] Let $f(z)$ and $g(z)$ be two transcendental entire functions of finite order, and $\alpha(z)(\not \equiv 0, \infty)$ be a small function with respect to both $f(z)$ and $g(z)$ with finitely many zeros. Suppose that $c$ is a non-zero complex constant, $n, k(\geq 0)$ and $m(\geq 1)$ are integers such that $n \geq 5 k+4 m+12$. If $\bar{E}_{2)}\left(\alpha(z),\left(f^{n}(z)\left(f^{m}(z)-\right.\right.\right.$ 1) $\left.f(z+c))^{(k)}\right)=\bar{E}_{2)}\left(\alpha(z),\left(g^{n}(z)\left(g^{m}(z)-1\right) g(z+c)\right)^{(k)}\right)$, then $f(z) \equiv \operatorname{tg}(z)$, where $t^{m}=1$. 
Since no attempts, till now, have so far been made by any researchers investigating the uniqueness problem by replacing $f(z+c)$ with $L_{c}(f)$ in all the above-stated Theorems $\mathrm{F}-\mathrm{K}$, it is, therefore, inevitable to ask the following question.

Question 1.1. What can be said about the uniqueness of $f$ and $g$ if one replace $f^{n}\left(f^{m}-1\right) f(z+c)$ by the difference polynomial $f(z)^{n} P(f(z)) L_{c}(f)$ in Theorems $F-K$ ?

In this paper, we paid our attention to the above question and proved the following three theorems that improve and extend Theorems I-K, respectively. Indeed, the following theorems are the main results of the paper.

Theorem 1.1. Let $f(z)$ and $g(z)$ be two transcendental entire functions of finite order, $\alpha(z)(\not \equiv 0, \infty)$ be a small function with respect to both $f(z)$ and $g(z)$. Suppose $c$ be a non-zero complex constant, $n, k(\geq 0), m(\geq k+1)$ are integers such that $n \geq$ $2 k+m+6$. If $\left(f(z)^{n} P(f(z)) L_{c}(f)\right)^{(k)}$ and $\left(g(z)^{n} P(g(z)) L_{c}(g)\right)^{(k)}$ share " $(\alpha(z), 2)$ ", then one of the following two conclusions can be realized.

(a) $f(z) \equiv \operatorname{tg}(z)$, where $t$ is a constant such that $t^{d}=1, d=\operatorname{gcd}\left(\lambda_{0}, \lambda_{1}, \ldots, \lambda_{m}\right)$, where $\lambda_{j}$ 's are defined by

$$
\lambda_{j}=\left\{\begin{array}{l}
n+1+j, \quad \text { if } a_{j} \neq 0 \\
n+1+m, \quad \text { if } a_{j}=0,
\end{array} \quad j=0,1, \ldots, m .\right.
$$

(b) $f$ and $g$ satisfy the algebraic equation $R\left(w_{1}, w_{2}\right)=0$, where $R\left(w_{1}, w_{2}\right)$ is given by $R\left(w_{1}, w_{2}\right)=w_{1}^{n} P\left(w_{1}\right) L_{c}\left(w_{1}\right)-w_{2}^{n} P\left(w_{2}\right) L_{c}\left(w_{2}\right)$.

Theorem 1.2. Let $f(z)$ and $g(z)$ be two transcendental entire functions of finite order, $\alpha(z)(\not \equiv 0, \infty)$ be a small function with respect to both $f(z)$ and $g(z)$. Suppose $c$ be a non-zero complex constant, $n, k(\geq 0), m(\geq k+1)$ are integers such that $n \geq 3 k+2 m+8$. If $\left(f(z)^{n} P(f(z)) L_{c}(f)\right)^{(k)}$ and $\left(g(z)^{n} P(g(z)) L_{c}(g)\right)^{(k)}$ share $(\alpha(z), 2)^{*}$, then the conclusions of Theorem 1.1 holds.

Theorem 1.3. Let $f(z)$ and $g(z)$ be two transcendental entire functions of finite order, $\alpha(z)(\not \equiv 0, \infty)$ be a small function with respect to both $f(z)$ and $g(z)$. Suppose $c$ be a non-zero complex constant, $n, k(\geq 0), m(\geq k+1)$ are integers such that $n \geq 9+$ $(7 k+5 m) / 2$. If $E_{2)}\left(\alpha(z),\left(f^{n} P(f(z)) L_{c}(f)\right)^{(k)}\right)=E_{2)}\left(\alpha(z),\left(g^{n}(z) P(g(z)) L_{c}(g)\right)^{(k)}\right)$, then the conclusions of Theorem 1.1 holds.

Remark 1.1. Clearly, for the particular choice of $c_{0}=0, L_{c}(f)$ becomes $f(z+c)$, and therefore Theorem 1.1 coincides with Theorem I and Theorem 1.2 with Theorem $J$.

Remark 1.2. In Theorem $K$, if one replace $\bar{E}_{2)}(\alpha(z), f(z))=\bar{E}_{2)}(\alpha(z), g(z))$ by $E_{2)}(\alpha(z), f(z))=E_{2)}(\alpha(z), g(z))$, for any two non-constant meromorphic functions $f$ and $g$, then the lower bound of $n$ can significantly reduced. Regarding this observation, we proved Theorem 1.3 .

Since for a particular choice of $c_{0}=-1, L_{c}(f)=\Delta_{c} f$, we observe the following corollaries.

Corollary 1.1. Let $f(z)$ and $g(z)$ be two transcendental entire functions of finite order, $\alpha(z)(\not \equiv 0, \infty)$ be a small function with respect to both $f(z)$ and $g(z)$. Suppose $c$ be a non-zero complex constant, $n, k(\geq 0), m(\geq k+1)$ are integers such that $n \geq$ $2 k+m+6$. If $\left(f(z)^{n} P(f(z)) \Delta_{c} f\right)^{(k)}$ and $\left(g(z)^{n} P(g(z)) \Delta_{c} g\right)^{(k)}$ share " $(\alpha(z), 2)$ ", then conclusion of Theorem 1.2 holds. 
Corollary 1.2. Let $f(z)$ and $g(z)$ be two transcendental entire functions of finite order, $\alpha(z)(\equiv \equiv, \infty)$ be a small function with respect to both $f(z)$ and $g(z)$. Suppose $c$ be a non-zero complex constant, $n, k(\geq 0), m(\geq k+1)$ are integers such that $n \geq$ $3 k+2 m+8$. If $\left(f(z)^{n} P(f(z)) \Delta_{c} f\right)^{(k)}$ and $\left(g(z)^{n} P(g(z)) \Delta_{c} g\right)^{(k)}$ share $(\alpha(z), 2)^{*}$, then the conclusions of Theorem 1.1 holds.

Corollary 1.3. Let $f(z)$ and $g(z)$ be two transcendental entire functions of finite order, $\alpha(z)(\equiv \equiv, \infty)$ be a small function with respect to both $f(z)$ and $g(z)$. Suppose $c$ be a non-zero complex constant, $n, k(\geq 0), m(\geq k+1)$ are integers such that $n \geq$ $9+(7 k+5 m) / 2$. If $E_{2)}\left(\alpha(z),\left(f^{n} P(f(z)) \Delta_{c} f\right)^{(k)}\right)=E_{2)}\left(\alpha(z),\left(g^{n}(z) P(g(z)) \Delta_{c} g\right)^{(k)}\right)$, then the conclusions of Theorem 1.1 holds.

\section{Some Lemmas}

We now prove several lemmas which will play key roles in proving the main results of the paper. Let $\mathcal{F}$ and $\mathcal{G}$ be two non-constant meromorphic functions. Henceforth we shall denote by $\mathcal{H}$ the following function

$$
H=\left(\frac{F^{\prime \prime}}{F^{\prime}}-\frac{2 F^{\prime}}{F-1}\right)-\left(\frac{G^{\prime \prime}}{G^{\prime}}-\frac{2 G^{\prime}}{G-1}\right) .
$$

Lemma 2.1. 2] Let $f(z)$ be a meromorphic function of finite order $\rho$, and let $c$ be a fixed non-zero complex constant. Then for each $\epsilon>0$, we have

$$
T(r, f(z+c))=T(r, f)+O\left(r^{\rho-1+\epsilon}\right)+O \log r .
$$

Lemma 2.2. 2] Let $f(z)$ be a meromorphic function of finite order $\rho$ and let $c$ be a non-zero complex number. Then for each $\epsilon>0$, we have

$$
m\left(r, \frac{f(z+c)}{f(z)}\right)+m\left(r, \frac{f(z)}{f(z+c)}\right)=O\left(r^{\rho-1+\epsilon}\right) .
$$

Lemma 2.3. [14 Let $f$ be a non-constant meromorphic function and let $\mathcal{R}(f)=$ $\sum_{i=0}^{n} a_{i} f^{i} / \sum_{j=0}^{m} b_{j} f^{j}$ be an irreducible rational function in $f$ with constant coefficients $\left\{a_{i}\right\}$ and $\left\{b_{j}\right\}$ where $a_{n} \neq 0$ and $b_{m} \neq 0$. Then

$$
T(r, \mathcal{R}(f))=d T(r, f)+S(r, f),
$$

where $d=\max \{n, m\}$.

Lemma 2.4. 9] If $N\left(r, 0 ; f^{(k)} \mid f \neq 0\right)$ denotes the counting function of those zeros of $f^{(k)}$ which are not the zeros of $f$, where a zero of $f^{(k)}$ is counted according to its multiplicity then

$$
N\left(r, 0 ; f^{(k)} \mid f \neq 0\right) \leq k \bar{N}(r, \infty ; f)+N(r, 0 ; f \mid<k)+k \bar{N}(r, 0 f \mid \geq k)+S(r, f) .
$$

Lemma 2.5. Let $F=f(z)^{n}(z) P(f(z)) L_{c}(f)$, where $f(z)$ is an entire function of finite order, and $f(z), f(z+c)$ share $0 C M$. Then

$$
T(r, F)=(n+m+1) T(r, f)+S(r, f) .
$$

Proof. Keeping in view of Lemmas 2.1 and 2.3, we have

$$
\begin{aligned}
T(r, F) & =T\left(r, f(z)^{n} P(f(z)) L_{c}(f)\right)=m\left(r, f^{n} P(f) L_{c}(f)\right) \\
& \leq m\left(r, f(z)^{n} P(f(z))\right)+m\left(r, L_{c}(f)\right)+S(r, f) \\
& \leq T\left(f(z)^{n} P(f(z))\right)+m\left(r, \frac{L_{c}(f)}{f(z)}\right)+m(r, f(z))+S(r, f) .
\end{aligned}
$$


i.e.,

$$
T(r, F) \leq(n+m+1) T(r, f)+S(r, f) .
$$

Since $f(z)$ and $f(z+c)$ share $0 \mathrm{CM}$, we must have $N\left(r, \infty ; \frac{L_{c}(f)}{f(z)}\right)=S(r, f)$. So, keeping in view of Lemmas 2.2 and 2.3. we have

$$
\begin{aligned}
& (n+m+1) T(r, f)=T\left(r, f(z)^{n+1} P(f(z))\right)=m\left(r, f(z)^{n+1} P(f(z))\right) \\
= & m\left(r, F \frac{f(z)}{L_{c}(f)}\right) \leq m(r, F)+m\left(r, \frac{f(z)}{L_{c}(f)}\right)+S(r, f) \\
\leq & T(r, F)+T\left(r, \frac{L_{c}(f)}{f(z)}\right)+S(r, f)=T(r, F)+N\left(r, \frac{L_{c}(f)}{f(z)}\right) \\
& +m\left(r, \frac{L_{c}(f)}{f(z)}\right)+S(r, f)=T(r, F)+S(r, f) .
\end{aligned}
$$

From the above two inequalities, we must have

$$
T(r, F)=(n+m+1) T(r, f)+S(r, f) .
$$

Lemma 2.6. 18 Let $f(z)$ and $g(z)$ be two non-constant meromorphic functions. Then

$N\left(r, \infty ; \frac{f}{g}\right)-N\left(r, \infty ; \frac{g}{f}\right)=N(r, \infty ; f)+N(r, 0 ; g)-N(r, \infty ; g)+N(r, 0 ; f)$.

Lemma 2.7. Let $f(z)$ be a transcendental entire function of finite order, $c \in \mathbb{C}-$ $\{0\}$ be finite complex constants and $n \in \mathbb{N}$. Let $F(z)=f(z)^{n} P(f(z)) L_{c}(f)$, where $L_{c}(f) \not \equiv 0$. Then we have

$$
(n+m) T(r, f) \leq T(r, F)-N\left(r, 0 ; L_{c}(f)\right)+S(r, f) .
$$

Proof. Using Lemmas 2.2 and 2.6, we get

$$
\begin{aligned}
& m\left(f(z)^{n+1} P(f(z))\right)=m\left(r, \frac{f(z) F}{L_{c}(f)}\right) \leq m(r, F)+m\left(r, \frac{f(z)}{L_{c}(f)}\right)+S(r, f) \\
\leq & m(r, F)+T\left(r, \frac{f(z)}{L_{c}(f)}\right)-N\left(r, \infty ; \frac{f(z)}{L_{c}(f)}\right)+S(r, f) \\
\leq & m(r, F)+T\left(r, \frac{L_{c}(f)}{f(z)}\right)-N\left(r, \infty ; \frac{f(z)}{L_{c}(f)}\right)+S(r, f) \\
\leq & m(r, F)+N\left(r, \infty ; \frac{L_{c}(f)}{f(z)}\right)+m\left(r, \frac{L_{c}(f)}{f(z)}\right)-N\left(r, \infty ; \frac{f(z)}{L_{c}(f)}\right)+S(r, f) \\
\leq & m(r, F)+N(r, 0 ; f)-N\left(r, 0 ; L_{c}(f)\right)+S(r, f) .
\end{aligned}
$$

i.e.,

$$
m\left(f(z)^{n+1} P(f(z))\right) \leq T(r, F)+T(r, f)-N\left(r, 0 ; L_{c}(f)\right)+S(r, f) .
$$

By Lemma 2.3, we get

$(n+m+1) T(r, f)=m\left(r, f^{n+1} P(f)\right) \leq T(r, F)+T(r, f)-N\left(r, 0 ; L_{c}(f)\right)+S(r, f)$. i.e.,

$$
(n+m) T(r, f) \leq T(r, F)-N\left(r, 0 ; L_{c}(f)\right)+S(r, f) .
$$


Lemma 2.8. 1] Let $F$ and $G$ be two non-constant meromorphic functions that share $(1,2)^{*}$. Then

$$
\begin{aligned}
& \bar{N}_{L}(r, 1 ; F)+\bar{N}_{F \geq 3}(r, 1 ; g \mid=1) \\
\leq & \bar{N}(r, 0 ; F)+N(r, \infty ; F)-\sum_{p=3}^{\infty} \bar{N}\left(r, 0 ; \frac{F^{\prime}}{F} \mid \geq p\right)-\bar{N}_{0}^{2}\left(r, 0 ; F^{\prime}\right)+S(r),
\end{aligned}
$$

where by $N_{0}^{2}\left(r, 0 ; F^{\prime}\right)$ is the counting function of those zeros of $F^{\prime}$ which are not the zeros of $F(F-1)$, where each simple zero is counted once and all other zeros are counted two times.

Lemma 2.9. Let $F$ and $G$ be two non-constant meromorphic functions such that $E_{2)}(1, F)=E_{2)}(1, G)$ and $H \not \equiv 0$. Then

$$
\begin{aligned}
& N(r, \infty ; H) \\
\leq & \bar{N}(r, 0 ; F \mid \geq 2)+\bar{N}(r, 0 ; G \mid \geq 2)+\bar{N}_{L}(r, 1 ; F)+\bar{N}_{L}(r, 1 ; G)+\bar{N}(r, \infty ; F \mid \geq 2) \\
& +\bar{N}(r, \infty ; G \mid \geq 2)+\bar{N}_{F \geq 3}(r, 1 ; F \mid G \neq 1)+\bar{N}_{G \geq 3}(r, 1 ; G \mid F \neq 1) \\
& +\bar{N}_{0}\left(r, 0 ; F^{\prime}\right)+\bar{N}_{0}\left(r, 0 ; G^{\prime}\right)+S(r, F)+S(r, G) .
\end{aligned}
$$

Proof. It can be easily verified that all possible poles of $H$ occur at (i) multiple zeros of $F$ and $G$, (ii) multiple poles of $F$ and $G$, (iii) the common zeros of $F-1$ and $G-1$ whose multiplicities are different, (iii) those 1-points of $F(G)$ which are not the 1-points of $F(G)$, (iv) zeros of $F^{\prime}$ which are not the zeros of $F(F-1)$, (v) zeros of $G^{\prime}$ which are not zeros of $G(G-1)$. Since all the poles of $H$ are simple the lemma follows from above. This proves the lemma.

Lemma 2.10. 1] If $f, g$ be share " $(1,1) "$ and $H \not \equiv 0$, then

$$
N(r, 1 ; f \mid \leq 1) \leq N(r, 0 ; H)+S(r, f) \leq N(r, \infty ; H)+S(r, f)+S(r, g) .
$$

Lemma 2.11. [1] If $f, g$ be two non-constant meromorphic functions such that $E_{1)}(1 ; f)=E_{1)}(1 ; g)$ and $H \not \equiv 0$, then

$$
N(r, 1 ; f \mid \leq 1) \leq N(r, 0 ; H) \leq N(r, \infty ; H)+S(r, f)+S(r, g) .
$$

Lemma 2.12. 1] If $f, g$ be share $(1,1)^{*}$ and $H \not \equiv 0$, then

$$
N^{E}(r, 1 ; f, g \mid \leq 1) \leq N(r, 0 ; H) \leq N(r, \infty ; H)+S(r, f)+S(r, g) .
$$

Lemma 2.13. 1] If $f, g$ be share $(1,1)^{*}$ and $H \not \equiv 0$, then

$$
\begin{aligned}
N(r, \infty ; H) \leq & \bar{N}(r, 0 ; f \mid \geq 2)+\bar{N}(r, 0 ; g \mid \geq 2)+\bar{N}(r, \infty ; f \mid \geq 2)+\bar{N}_{*}(r, 1 ; f, g) \\
& +\bar{N}(r, \infty ; g \mid \geq 2)+\bar{N}_{0}\left(r, 0 ; f^{\prime}\right)+\bar{N}_{0}\left(r, 0 ; g^{\prime}\right)+S(r, f)+S(r, g),
\end{aligned}
$$

where $\bar{N}_{0}\left(r, 0 ; f^{\prime}\right)$ is the reduced counting function of those zeros of $f^{\prime}$ which are not the zeros of $f(f-1)$ and $\bar{N}_{0}\left(r, 0 ; g^{\prime}\right)$ is similarly defined.

Lemma 2.14. 1] Let $E_{2)}(1 ; f)=E_{2)}(1 ; g)$. Then

$$
\begin{aligned}
& \bar{N}_{f \geq 3}(r, 1 ; f \mid g \neq 1) \\
\leq & \frac{1}{2} \bar{N}(r, 0 ; f)+\frac{1}{2} \bar{N}(r, \infty ; f)-\frac{1}{2} \sum_{p=3}^{\infty} \bar{N}\left(r, 0 ; \frac{f^{\prime}}{F} \mid \geq p\right)-\frac{1}{2} \bar{N}_{0}^{2}\left(r, 0 ; f^{\prime}\right)+S(r),
\end{aligned}
$$


Lemma 2.15. 22] Let $f$ be a non-constant meromorphic function, and $p, k$ be positive integers. Then

$$
\begin{gathered}
N_{p}\left(r, 0 ; f^{(k)}\right) \leq T\left(r, f^{(k)}-T(r, f)+N_{p+k}(r, 0 ; f)+S(r, f),\right. \\
N_{p}\left(r, 0 ; f^{(k)}\right) \leq k \bar{N}(r, \infty ; f)+N_{p+k}(r, 0 ; f)+S(r, f) .
\end{gathered}
$$

\section{Proofs of the theorems}

Proof of Theorem 1.1. Let $F=\frac{F_{1}^{(k)}}{\alpha(z)}$ and $G=\frac{G_{1}^{(k)}}{\alpha(z)}$, where $F_{1}=f^{n}(z) P(f(z)) L_{c}(f)$ and $G_{1}=\left(g^{n}(z) P(g(z)) L_{c}(g)\right)$. Then $F$ and $G$ are two transcendental meromorphic functions that share " $(1,2)$ " except the zeros and poles of $\alpha(z)$. We consider the following two cases.

Case 1: Suppose $H \not \equiv 0$. Since $F$ and $G$ share " $(1,2)$ ", it follows that $F$ and $G$ share $(1,1)^{*}$. Keeping in view of Lemmas 2.10 and 2.13, we see that

$$
\begin{aligned}
\bar{N}(r, 1 ; F)= & N(r, 1 ; F \mid \leq 1)+\bar{N}(r, 1 ; F \mid \geq 2) \leq N(r, \infty ; H)+\bar{N}(r, 1 ; F \mid \geq 2) \\
\leq & \bar{N}(r, 0 ; F \mid \geq 2)+\bar{N}(r, 0 ; G \mid \geq 2)+\bar{N}_{*}(r, 1 ; F, G)+\bar{N}(r, 1 ; F \mid \geq 2) \\
& \bar{N}_{0}\left(r, 0 ; F^{\prime}\right)+\bar{N}_{0}\left(r, 0 ; G^{\prime}\right)+S(r, F)+S(r, G) .
\end{aligned}
$$

Since $F, G$ share "(1,2)", we must have $\bar{N}_{F>2}(r, 1 ; F \mid G \neq 1)=S(r, F)$ and $\bar{N}(r, 1 ; F|\geq 2, G|=1)=S(r, F)$. Therefore, keeping in view of the above observation and Lemma 2.4, we get

$$
\begin{aligned}
& \bar{N}_{0}\left(r, 0 ; G^{\prime}\right)+\bar{N}(r, 1 ; F \mid \geq 2)+\bar{N}_{*}(r, 1 ; F, G) \\
\leq & \bar{N}_{0}\left(r, 0 ; G^{\prime}\right)+\bar{N}(r, 1 ; F \mid \geq 3)+\bar{N}_{F \geq 2}(r, 1 ; F \mid G \neq 1)+\bar{N}(r, 1 ; F|\geq 2, G|=1) \\
& +\bar{N}(r, 1 ; F|\geq 2, G| \geq 2)+S(r, G) \\
\leq & \bar{N}_{0}\left(r, 0 ; G^{\prime}\right)+\bar{N}(r, 1 ; G \mid \geq 3)+\bar{N}(r, 1 ; G \mid \geq 2)+S(r, F)+S(r, G) \\
\leq & N\left(r, 0 ; G^{\prime} \mid G \neq 0\right) \leq \bar{N}(r, 0 ; G)+S(r, G) .
\end{aligned}
$$

Hence using (3.1), (3.2), Lemmas 2.2, 2.7 and 2.15, we get from second fundamental theorem that

$$
\begin{aligned}
& (n+m) T(r, f) \leq T\left(r, F_{1}\right)-N\left(r, 0 ; L_{c}(f)\right)+S(r, f) \\
\leq & T(r, F)+N_{k+2}\left(r, 0 ; F_{1}\right)-N_{2}(r, 0 ; F)-N\left(r, 0 ; L_{c}(f)\right)+S(r, f) \\
\leq & \bar{N}(r, 0 ; F)+\bar{N}(r, 1 ; F)+\bar{N}(r, \infty ; F)+N_{k+2}\left(r, 0 ; F_{1}\right)-N_{2}(r, 0 ; F) \\
& -N\left(r, 0 ; L_{c}(f)\right)-\bar{N}_{0}\left(r, 0 ; F^{\prime}\right)+S(r, f) \\
\leq & \bar{N}(r, 0 ; F)+\bar{N}(r, 0 ; F \mid \geq 2)+\bar{N}(r, 0 ; G \mid \geq 2)+\bar{N}(r, 1 ; F \mid \geq 2)+\bar{N}_{L}(r, 1 ; F) \\
& +\bar{N}_{L}(r, 1 ; G)+\bar{N}_{0}\left(r, 0 ; G^{\prime}\right)+N_{k+2}\left(r, 0 ; F_{1}\right)-N_{2}(r, 0 ; F)-N\left(r, 0 ; L_{c}(f)\right) \\
\leq & N_{k+2}\left(r, 0 ; F_{1}\right)+N_{2}(r, 0 ; G)-N\left(r, 0 ; L_{c}(f)\right)+S(r, f)+S(r, g) \\
\leq & N_{k+2}\left(r, 0 ; f(z)^{n} P(f(z)) L_{c}(f)\right)+N_{k+2}\left(r, 0 ; g(z)^{n} P(g(z)) L_{c}(g)\right) \\
& -N\left(r, 0 ; L_{c}(f)\right)+S(r, f)+S(r, g) \\
\leq & N_{k+2}\left(r, 0 ; f^{n}(z)\right)+N_{k+2}(r, 0 ; P(f))+N_{k+2}\left(r .0 ; g^{n}(z)\right)+N_{k+2}(r, 0 ; P(g)) \\
& +N\left(r, 0 ; L_{c}(g)\right)+S(r, f)+S(r, g) \\
\leq & (k+2) \bar{N}(r, 0 ; f)+N(r, 0 ; P(f))+(k+2) \bar{N}(r, 0 ; g)+N(r, 0 ; P(g)) \\
& +N\left(r, 0 ; L_{c}(f)\right)+S(r, f)+S(r, g) \\
\leq & (k+m+1)(T(r, f)+T(r, g))+T\left(r, L_{c}(g)\right)+S(r, f)+S(r, g) .
\end{aligned}
$$


i.e.,

$$
\begin{aligned}
& (n+m) T(r, f) \\
\leq & (k+m+1)(T(r, f)+T(r, g))+m\left(r, L_{c}(g)\right)+S(r, f)+S(r, g) \\
\leq & (k+m+1)(T(r, f)+T(r, g))+m\left(r, \frac{L_{c}(g)}{g}\right)+m(r, g)+S(r, f)+S(r, g) \\
\leq & (k+m+1)(T(r, f)+T(r, g))+T(r, g)+S(r, f)+S(r, g) .
\end{aligned}
$$

In a similarly, we get

$$
(n+m) T(r, g) \leq(k+m+1)(T(r, f)+T(r, g))+T(r, f)+S(r, f)+S(r, g) .
$$

Combining (3.3) and (3.4), we get

$$
(n-2 k-m-5)(T(r, f)+T(r, g)) \leq S(r, f)+S(r, g),
$$

which is a contradicts with $n \geq 2 k+m+6$.

Case 2: Suppose $H \equiv 0$. Then by integration we get

$$
F=\frac{A G+B}{C G+D}
$$

where $A, B, C, D$ are complex constant satisfying $A D-B C \neq 0$.

Subcase 2.1: Suppose $A C \neq 0$. Then $F-\frac{A}{C}=\frac{-(A D-B C)}{C(C G+D)} \neq 0$. So $F$ omits the value $\frac{A}{C}$.

Therefore, by Lemma 2.7 and the Second Fundamental Theorem of Nevalinna, we get

$$
\begin{aligned}
& (n+m) T(r, f) \leq T\left(r, f(z)^{n} P(f(z)) L_{c}(f)\right)-N\left(r, 0 ; L_{c}(f)\right)+S(r, f) \\
\leq \quad & T\left(r, F_{1}\right)-N\left(r, 0 ; L_{c}(f)\right)+S(r, f) \leq T(r, F)+N_{k+1}\left(r, 0 ; F_{1}\right)-\bar{N}(r, 0 ; F) \\
& -N\left(r, 0 ; L_{c}(f)\right)+S(r, f) \leq \bar{N}(r, 0 ; F)+\bar{N}(r, \infty ; F)+\bar{N}\left(r, \frac{A}{C} ; F\right) \\
& -N\left(r, 0 ; L_{c}(f)\right)+N_{k+1}\left(r, 0 ; F_{1}\right)-N(r, 0 ; F)+S(r, f) \\
\leq \quad & N_{k+1}\left(r, 0 ; f^{n} P(f) L_{c}(f)\right)-N\left(r, 0 ; L_{c}(f)\right)+S(r, f) \leq(k+1) \bar{N}(r, 0 ; f) \\
& +N(r, 0 ; P(f))+S(r, f) \leq(k+m+1) T(r, f)+S(r, f),
\end{aligned}
$$

which is a contradicts with $n \geq 2 k+m+6$.

Subcase 2.2: Suppose $A C=0$. Since $A D-B C \neq 0, A$ and $C$ both can not be simultaneously zero.

Subcase 2.2.1: Let $A \neq 0$ and $C=0$. Then (3.5) becomes $F=A_{1} G+B_{1}$, where $A_{1}=A / D$ and $B_{1}=B / D$. If $f$ has no 1-point, then by Lemma 2.7 and the second fundamental theorem of Nevallina, we get

$$
\begin{aligned}
& (n+m) T(r, f) \leq T\left(r, f(z)^{n} P(f(z)) L_{c}(f)\right)-N\left(r, 0 ; L_{c}(f)\right)+S(r, f) \\
\leq \quad & T\left(r, F_{1}\right)-N\left(r, 0 ; L_{c}(f)\right)+S(r, f) \leq T(r, F)+N_{k+1}\left(r, 0 ; F_{1}\right)-\bar{N}(r, 0 ; F) \\
& -N\left(r, 0 ; L_{c}(f)\right)+S(r, f) \leq \bar{N}(r, 0 ; F)+\bar{N}(r, \infty ; F)+\bar{N}(r, 1 ; F) \\
& +N_{k+1}\left(r, 0 ; F_{1}\right)-\bar{N}(r, 0 ; F)-N\left(r, 0 ; L_{c}(f)\right)+S(r, f) \\
\leq & \bar{N}_{k+1}\left(r, 0 ; f^{n} P(f) L_{c}(f)\right)-N\left(r, 0 ; L_{c}(f)\right) \leq(k+m+1) T(r, f)+S(r, f),
\end{aligned}
$$

which is a contradiction since $n \geq 2 k+m+6$. Let $f$ has some 1-point. Then $A_{1}+B_{1}=1$. Therefore, $F=A_{1} G+1-A_{1}$. If $A_{1} \neq 1$, then using Lemmas 2.7 
2.5. 2.15 and the second fundamental theorem, we get

$$
\begin{aligned}
& (n+m) T(r, g) \leq T\left(r, g^{n} P(g) L_{c}(g)\right)-N\left(r, 0 ; L_{c}(g)\right)+S(r, g) \\
\leq & T\left(r, G_{1}\right)-N\left(r, 0 ; L_{c}(g)\right)+S(r, g) \\
\leq & T(r, G)+N_{k+1}\left(r, 0 ; G_{1}\right)-\bar{N}(r, 0 ; G)-N\left(r, 0 ; L_{c}(g)\right)+S(r, g) \\
\leq & \bar{N}(r, 0 ; G)+\bar{N}(r, \infty ; G)+\bar{N}\left(r, \frac{1-A_{1}}{A_{1}} ; G\right)+N_{k+1}\left(r, 0 ; G_{1}\right)-\bar{N}(r, 0 ; G) \\
& -N\left(r, 0 ; L_{c}(g)\right)+S(r, g) \\
\leq & N_{k+1}\left(r, 0 ; G_{1}\right)+\bar{N}(r, 0 ; F)-N\left(r, 0 ; L_{c}(g)\right)+S(r, g) \\
\leq & N_{k+1}\left(r, 0 ; F_{1}\right)+N_{k+1}\left(r, 0 ; G_{1}\right)-N\left(r, 0 ; L_{c}(g)\right)+S(r, g) \\
\leq & (k+m+1) T(r, f)+T\left(r, L_{c}(f)\right)+(k+m+1) T(r, g)+S(r, f)+S(r, g) \\
\leq & (2 k+2 m+3) T(r, g)+S(r, g) .
\end{aligned}
$$

i.e.,

$$
(n-2 k-m-3) T(r, g) \leq S(r, g),
$$

which is a contradiction since $n \geq 2 k+m+6$. Hence $A_{1}=1$, and therefore we have $F \equiv G$. i.e.,

$$
\left(f(z)^{n} P(f(z)) L_{c}(f)\right)^{(k)} \equiv\left(g(z)^{n} P(g(z)) L_{c}(g)\right)^{(k)} .
$$

Integrating $k$ times we get

$$
f^{n} P(f) L_{c}(f) \equiv g^{n} P(g) L_{c}(g)+p(z),
$$

where $p(z)$ is a polynomial of degree at most $k-1$. Suppose $p(z) \not \equiv 0$. Then from (3.6), we have

$$
\frac{f^{n} P(f) L_{c}(f)}{p(z)} \equiv \frac{g^{n} P(g) L_{c}(g)}{p(z)}+1 .
$$

Now, using Lemmas 2.2, 2.6 and the second fundamental theorem, we get

$$
\begin{aligned}
& (n+m) T(r, f) \leq T\left(r, f^{n} P(f) L_{c}(f)\right)-N\left(r, 0 ; L_{c}(f)\right)+S(r, f) \\
\leq & T\left(r, f^{n} P(f) L_{c}(f) / p(z)\right)-N\left(r, 0 ; L_{c}(f)\right)+S(r, f) \\
\leq & \bar{N}\left(r, 0 ; \frac{f^{n} P(f) L_{c}(f)}{p}\right)+\bar{N}\left(r, \infty ; \frac{f^{n} P(f) L_{c}(f)}{p}\right)+\bar{N}\left(r, 1 ; \frac{f^{n} P(f) L_{c}(f)}{p}\right) \\
& -N\left(r, 0 ; L_{c}(f)\right)+S(r, f) \\
\leq & \bar{N}(r, 0 ; f)+\bar{N}(r, 0 ; P(f))+\bar{N}\left(r, 0 ; \frac{g^{n} P(g) L_{c}(g)}{p}\right)+S(r, f) \\
\leq & \bar{N}(r, 0 ; f)+\bar{N}(r, 0 ; P(f))+\bar{N}(r, 0 ; g)+\bar{N}(r, 0 ; P(g))+\bar{N}\left(r, 0 ; L_{c}(g)\right)+S(r, f) \\
\leq & (m+1) T(r, f)+(m+1) T(r, g)+T\left(r, L_{c}(g)\right)+S(r, f)+S(r, g) \\
\leq & (m+1) T(r, f)+(m+1) T(r, g)+m\left(r, \frac{L_{c}(g)}{g}\right)+m(r, g)+S(r, f)+S(r, g) \\
\leq & (m+1) T(r, f)+(m+2) T(r, g)+S(r, f)+S(r, g) .
\end{aligned}
$$

Similarly, we get

$$
(n+m) T(r, g) \leq(m+1) T(r, g)+(m+2) T(r, f)+S(r, f)+S(r, g) .
$$

Combining the above two equations, we get

$$
(n-m-3)(T(r, f)+T(r, g)) \leq S(r, f)+S(r, g),
$$


G. HALDAR

which contradicts to the fact that $n \geq 2 k+m+6$. Hence $p(z) \equiv 0$ and so from (3.6), we obtain

$$
f^{n} P(f) L_{c}(f) \equiv g^{n} P(g) L_{c}(g) .
$$

i.e.

$$
\begin{aligned}
& f^{n}\left(a_{m} f^{m}+a_{m-1} f^{m-1}+\ldots+a_{1} f+a_{0}\right)\left(f(z+c)+c_{0} f(z)\right) \\
\equiv & g^{n}\left(a_{m} g^{m}+a_{m-1} g^{m-1}+\ldots+a_{1} g+a_{0}\right)\left(g(z+c)+c_{0} g(z)\right) .
\end{aligned}
$$

Let $h=f / g$. Then the above equation can be written as

$$
\begin{aligned}
& {\left[a_{m}\left(h^{n+m} h(z+c)-1\right) g^{m}+a_{m-1}\left(h^{n+m-1} h(z+c)-1\right) g^{m-1}+\ldots\right.} \\
& \left.+a_{0}\left(h^{n} h(z+c)-1\right)\right] g(z+c) \\
& \equiv-c_{0}\left[a_{m}\left(h^{n+m+1}-1\right) g^{m}+a_{m-1}\left(h^{n+m}-1\right) g^{m-1}+\ldots+a_{0}\left(h^{n+1}-1\right)\right] g(z) .
\end{aligned}
$$

If $h$ is constant, then the above equation can be written as

$$
\left[a_{m}\left(h^{n+m+1}-1\right) g^{m}+a_{m-1}\left(h^{n+m}-1\right) g^{m-1}+\ldots+a_{0}\left(h^{n+1}-1\right)\right] L_{c}(g) \equiv 0 .
$$

Since $L_{c}(g) \not \equiv 0$, we must have

$$
a_{m}\left(h^{n+m+1}-1\right) g^{m}+a_{m-1}\left(h^{n+m}-1\right) g^{m-1}+\ldots+a_{0}\left(h^{n+1}-1\right)=0 .
$$

Then by a similar argument as in the Case 2 in the proof of Theorem 11 [17, we obtain $f=t g$, where $t$ a constant such that $t^{d}=1, d=\operatorname{gcd}\left(\lambda_{0}, \lambda_{1}, \ldots, \lambda_{m}\right)$, where $\lambda_{j}$ 's are defined by

$$
\lambda_{j}=\left\{\begin{array}{l}
n+1+j, \quad \text { if } a_{j} \neq 0 \\
n+1+m, \text { if } a_{j}=0,
\end{array} \quad j=0,1, \ldots, m\right.
$$

If $h$ is not constant, then it follows that $f, g$ satisfy the algebraic equation $R\left(w_{1}, w_{2}\right)=0$, where

$$
R\left(w_{1}, w_{2}\right)=w_{1}^{n} P\left(w_{1}\right) L_{c}\left(w_{1}\right)-w_{2}^{n} P\left(w_{2}\right) L_{c}\left(w_{2}\right) .
$$

Subcase 2.2.2: Let $A=0$ and $C \neq 0$. Then (3.5) becomes

$$
F=\frac{1}{A_{2} G+B_{2}},
$$

where $A_{2}=C / B$ and $B_{2}=D / B$. If $F$ has no 1-point, then by a similar argument as done in Subcase 2.2.1, we can get a contradiction. Let $F$ has some 1-point. Then $A_{2}+B_{2}=1$. If $A_{2} \neq 1$, then (3.9) can be written as

$$
F=\frac{1}{A_{2} G+1-A_{2}} .
$$

Since $F$ is entire and $A_{2} \neq 0, G$ omits the value $\left(1-A_{2}\right) / A_{2}$. Therefore, by Lemma 2.7 and the second fundamental theorem, we get

$$
\begin{aligned}
& (n+m) T(r, g) \leq T\left(r, g^{n} P(g) L_{c}(g)\right)-N\left(r, 0 ; L_{c}(g)\right)+S(r, g) \\
\leq & T\left(r, G_{1}\right)-N\left(r, 0 ; L_{c}(g)\right)+S(r, g) \\
\leq & T(r, G)+N_{k+1}\left(r, 0 ; G_{1}\right)-\bar{N}(r, 0 ; G)-N\left(r, 0 ; L_{c}(g)\right)+S(r, g) \\
\leq & \bar{N}(r, 0 ; G)+\bar{N}(r, \infty ; G)+\bar{N}\left(r, \frac{1-A_{2}}{A_{2}} ; G\right)+N_{k+1}\left(r, 0 ; G_{1}\right)-\bar{N}(r, 0 ; G) \\
& -N\left(r, 0 ; \Delta_{c} g\right)+S(r, g) .
\end{aligned}
$$


i.e.,

$$
\begin{aligned}
& (n+m) T(r, g) \leq N_{k+1}\left(r, 0 ; g^{n} P(g) L_{c}(g)\right)-N\left(r, 0 ; L_{c}(g)\right)+S(r, g) \\
\leq \quad & N_{k+1}\left(r, 0 ; g^{n}\right)+N_{k+1}(r, 0 ; P(g))+S(r, g) \\
\leq \quad & (k+1) \bar{N}(r, 0 ; g)+N(r, 0 ; P(g))+S(r, g) \leq(k+m+1) T(r, g)+S(r, g),
\end{aligned}
$$

which is a contradiction since $n \geq 2 k+m+6$. Hence $A_{2}=1$. So, from (3.10), we get $F G \equiv 1$. i.e.,

$$
\left(f(z)^{n} P(f(z)) L_{c}(f)\right)^{(k)}\left(g(z)^{n} P(g(z)) L_{c}(g)\right)^{(k)} \equiv \alpha^{2}(z) .
$$

Let $u_{1}, u_{2}, \ldots, u_{t}, 1 \leq t \leq m$ be the distinct zeros of $P(z)$. Since $m \geq k+1, a_{0} \neq 0$ and $f$ is entire, it is easily seen from (3.11) that $f$ has atleast two finite Picard exceptional values, which is not possible. Hence the proof is complete.

Proof of Theorem 1.2. Let $F$ and $G$ be defined as in Theorem 1.1. Then $F$ and $G$ are two transcendental meromorphic functions that share $(1,2)^{*}$ except the zeros and poles of $\alpha(z)$. We consider the following two cases.

Case 1: Suppose $H \not \equiv 0$. Since $F$ and $G$ share $(1,2)^{*}$, it follows that $F$ and $G$ share $(1,1)^{*}$. Also we note that $\bar{N}(r, 1 ; F|=1, G|=0)=S(r, F)+S(r, G)$. Keeping in view of Lemmas 2.12 and 2.13, we see that

$$
\begin{aligned}
& \bar{N}(r, 1 ; F)=N(r, 1 ; F \mid \leq 1)+\bar{N}(r, 1 ; F \mid \geq 2) \\
\leq & \bar{N}(r, 1 ; F|=1, G|=0)+\bar{N}^{E}(r, 1 ; F, G \mid \leq 1)+\bar{N}(r, 1 ; F \mid \geq 2) \\
\leq & N(r, \infty ; H)+\bar{N}(r, 1 ; F \mid \geq 2)+S(r, F)+S(r, G) \\
\leq & \bar{N}(r, 0 ; F \mid \geq 2)+\bar{N}(r, 0 ; G \mid \geq 2)+\bar{N}_{*}(r, 1 ; F, G)+\bar{N}(r, 1 ; F \mid \geq 2) \\
& \bar{N}_{0}\left(r, 0 ; F^{\prime}\right)+\bar{N}_{0}\left(r, 0 ; G^{\prime}\right)+S(r, F)+S(r, G) .
\end{aligned}
$$

Since $F, G$ share $(1,2)^{*}$, we must have $\bar{N}_{F \geq 2}(r, 1 ; F \mid G \neq 1)=S(r, F)+S(r, G)$, $\bar{N}(r, 1 ; F|=2, G|=1)=S(r, F)+S(r, G)$. Therefore, using Lemma 2.8, we get

$$
\begin{aligned}
& \bar{N}(r, 1 ; F \mid \geq 2) \\
\leq & \bar{N}_{F \geq 2}(r, 1 ; F \mid G \neq 1)+\bar{N}(r, 1 ; F|\geq 2, G|=1)+\bar{N}(r, 1 ; F|\geq 2, G| \geq 2) \\
\leq & \bar{N}_{F \geq 2}(r, 1 ; F \mid G \neq 1)+\bar{N}(r, 1 ; F|=2, G|=1)+\bar{N}_{F \geq 3}(r, 1 ; G \mid=1) \\
& +\bar{N}(r, 1 ; G \mid \geq 2)+S(r, F)+S(r, G) \\
\leq & \bar{N}(r, 0 ; F)+\bar{N}(r, 1 ; G \mid \geq 2)+S(r, F)+S(r, G) .
\end{aligned}
$$

Again using (3.13) and Lemma 2.4, we get

$$
\begin{aligned}
& \bar{N}_{0}\left(r, 0 ; G^{\prime}\right)+\bar{N}(r, 1 ; F \mid \geq 2)+\bar{N}_{*}(r, 1 ; F, G) \\
\leq & \bar{N}_{0}\left(r, 0 ; G^{\prime}\right)+\bar{N}(r, 1 ; G \mid \geq 2)+\bar{N}(r, 1 ; G \mid \geq 3)+\bar{N}(r, 0 ; F)+S(r, G) \\
\leq & \bar{N}_{0}\left(r, 0 ; G^{\prime}\right)+N(r, 1 ; G)-\bar{N}(r, 1 ; G)+\bar{N}(r, 0 ; F)+S(r, F)+S(r, G) \\
\leq & N\left(r, 0 ; G^{\prime} \mid G \neq 0\right)+\bar{N}(r, 0 ; F)+S(r, F)+S(r, G) \\
\leq & \bar{N}(r, 0 ; F)+\bar{N}(r, 0 ; G)+S(r, F)+S(r, G) .
\end{aligned}
$$

Hence using (3.12), (3.14), Lemmas 2.2) and 2.7, Second fundamental theorem of Nevalinna, we get

$$
\begin{aligned}
& (n+m) T(r, f) \leq T\left(r, f^{n} P(f) L_{c}(f)\right)-N\left(r, 0 ; L_{c}(f)\right)+S(r, f) \\
\leq & T(r, F)+N_{k+2}\left(r, 0 ; F_{1}\right)-N_{2}(r, 0 ; F)-N\left(r, 0 ; L_{c}(f)\right)+S(r, f) .
\end{aligned}
$$


i.e.,

$$
\begin{aligned}
& (n+m) T(r, f) \leq \bar{N}(r, 0 ; F)+\bar{N}(r, \infty ; F)+\bar{N}(r, 1 ; F)-\bar{N}\left(r, 0 ; F^{\prime}\right) \\
& +N_{k+2}\left(r, 0 ; F_{1}\right)-N_{2}(r, 0 ; F)-N\left(r, 0 ; L_{c}(f)\right)+S(r, f) \\
\leq \quad & N_{2}(r, 0 ; F)+N_{2}(r, 0 ; G)+\bar{N}(r, 0 ; F)+N_{k+2}\left(r, 0 ; F_{1}\right)-N_{2}(r, 0 ; F) \\
& -N\left(r, 0 ; L_{c}(f)\right)+S(r, f)+S(r, g) \\
\leq \quad & N_{k+2}\left(r, 0 ; F_{1}\right)+N_{k+2}\left(r, 0 ; G_{1}\right)+N_{k+1}\left(r, 0 ; F_{1}\right)-N\left(r, 0 ; L_{c}(f)\right) \\
& +S(r, f)+S(r, g) \leq(k+2)(\bar{N}(r, 0 ; f)+\bar{N}(r, 0 ; g))+N(r, 0 ; P(f)) \\
& +N(r, 0 ; P(g))+N(r, 0 ; P(f))+N\left(r, 0 ; L_{c}(g)\right)+(k+1) \bar{N}(r, 0 ; f) \\
& +N\left(r, 0 ; L_{c}(f)\right)+S(r, f)+S(r, g) \leq(k+m+2)(T(r, f)+T(r, g)) \\
& +(k+m+1) T(r, f)+T\left(r, L_{c}(f)\right)+T\left(r, L_{c}(g)\right)+S(r, f)+S(r, g) \\
\leq \quad & (k+m+2)(T(r, f)+T(r, g))+(k+m+1) T(r, f)+m\left(r, \frac{L_{c}(f)}{f}\right) \\
& +m(r, f)+m\left(r, \frac{L_{c}(g)}{g}\right)+m(r, g)+S(r, f)+S(r, g) \\
\leq \quad & (k+m+3)(T(r, f)+T(r, g))+(k+m+1) T(r, f) \\
& +S(r, f)+S(r, g) .
\end{aligned}
$$

In a similar manner, we obtain

$$
\begin{aligned}
(n+m) T(r, g) \leq & (k+m+3)(T(r, f)+T(r, g))+(k+m+1) T(r, g) \\
& +S(r, f)+S(r, g) .
\end{aligned}
$$

Combining (3.15) and (3.16), we get

$$
(n-3 k-2 m+7)(T(r, f)+T(r, g)) \leq S(r, f)+S(r, g),
$$

which is a contradicts to the fact that $n \geq 3 k+2 m+8$.

Case 2: Let $H \equiv 0$. This case can be carried out similarly as done in case 2 of the proof of Theorem 1.1. So, we omit the details. This proves Theorem 1.2 .

Proof of Theorem 1.3. Let $F$ and $G$ be defined as in Theorem 1.1. Then $F$ and $G$ are transcendental meromorphic functions such that $E_{2)}(1, F)=E_{2)}(1, G)$ except the zeros and poles of $\alpha(z)$. Let us discuss the following two cases.

Case 1: Let $H \not \equiv 0$. Since $E_{2)}(1, F)=E_{2)}(1, G)$, it follows that $E_{1)}(1, F)=$ $E_{1)}(1, G)$. Keeping in view of Lemmas 2.9, 2.11 and 2.14, we see that

$$
\begin{aligned}
& \bar{N}(r, 1 ; F)=N(r, 1 ; F \mid \leq 1)+\bar{N}(r, 1 ; F \mid \geq 2) \\
\leq & N(r, H)+\bar{N}(r, 1 ; F \mid=2)+\bar{N}_{F \geq 3}(r, 1 ; F \mid G \neq 1)+\bar{N}(r, 1 ; F|\geq 3, G| \geq 3) \\
\leq & N(r, \infty ; H)+\bar{N}(r, 1 ; G \mid=2)+\bar{N}(r, 1 ; G \mid \geq 3)+\bar{N}_{F \geq 3}(r, 1 ; F \mid G \neq 1) \\
& +S(r, F)+S(r, G) \\
\leq & N(r, \infty ; H)+\bar{N}(r, 1 ; G \mid \geq 2)+\bar{N}_{F \geq 3}(r, 1 ; F \mid G \neq 1)+S(r, F)+S(r, G) \\
\leq & \bar{N}(r, 0 ; F \mid \geq 2)+\bar{N}(r, 0 ; G \mid \geq 2)+\bar{N}_{L}(r, 1 ; F)+\bar{N}_{L}(r, 1 ; G)+\bar{N}(r, 1 ; G \mid \geq 2) \\
& +2 \bar{N}_{F \geq 3}(r, 1 ; F \mid G \neq 1)+\bar{N}_{G \geq 3}(r, 1 ; G \mid F \neq 1)+\bar{N}_{0}\left(r, 0 ; F^{\prime}\right)+\bar{N}_{0}\left(r, 0 ; G^{\prime}\right) \\
& +S(r, F)+S(r, G) .
\end{aligned}
$$


i.e.,

$$
\begin{aligned}
& \bar{N}(r, 1 ; F) \\
\leq & \bar{N}(r, 0 ; F \mid \geq 2)+\bar{N}(r, 0 ; G \mid \geq 2)+\bar{N}_{L}(r, 1 ; F)+\bar{N}_{L}(r, 1 ; G)+\bar{N}(r, 1 ; G \mid \geq 2) \\
& +2 \bar{N}_{F \geq 3}(r, 1 ; F \mid G \neq 1)+\bar{N}_{G \geq 3}(r, 1 ; G \mid F \neq 1)+\bar{N}_{0}\left(r, 0 ; F^{\prime}\right)+\bar{N}_{0}\left(r, 0 ; G^{\prime}\right) \\
& +S(r, F)+S(r, G) \\
\leq & \bar{N}(r, 0 ; F \mid \geq 2)+\bar{N}(r, 0 ; G \mid \geq 2)+\bar{N}_{L}(r, 1 ; F)+\bar{N}_{L}(r, 1 ; G)+\bar{N}(r, 1 ; G \mid \geq 2) \\
+ & \bar{N}(r, 0 ; F)+\frac{1}{2} \bar{N}(r, 0 ; G)+\bar{N}_{0}\left(r, 0 ; F^{\prime}\right)+\bar{N}_{0}\left(r, 0 ; G^{\prime}\right)+S(r, F)+S(r, G \nmid 3.17)
\end{aligned}
$$

Now using Lemma 2.4, we get

$$
\begin{aligned}
& \bar{N}_{0}\left(r, 0 ; G^{\prime}\right)+\bar{N}(r, 1 ; G \mid \geq 2)+\bar{N}_{L}(r, 1 ; F)+\bar{N}_{L}(r, 1 ; G) \\
\leq & \bar{N}_{0}\left(r, 0 ; G^{\prime}\right)+\bar{N}(r, 1 ; G \mid \geq 2)+\bar{N}(r, 1 ; G \mid \geq 3)+S(r, G) \\
\leq & \bar{N}_{0}\left(r, 0 ; G^{\prime}\right)+N(r, 1 ; G)-\bar{N}(r, 1 ; G)+S(r, F)+S(r, G) \\
\leq & N\left(r, 0 ; G^{\prime} \mid G \neq 0\right) \leq \bar{N}(r, 0 ; G)+S(r, F)+S(r, G) .
\end{aligned}
$$

Therefore, using (3.17), (3.18), Lemmas 2.2 and 2.7 we get from Second Fundamental Theorem that

$$
\begin{aligned}
& (n+m) T(r, f) \leq T\left(r, f^{n} P(f) L_{c}(f)\right)-N\left(r, 0 ; L_{c}(f)\right)+S(r, f) \\
\leq \quad & T(r, F)+N_{k+2}\left(r, 0 ; F_{1}\right)-N_{2}(r, 0 ; F)-N\left(r, 0 ; L_{c}(f)\right)+S(r, f) \\
\leq \quad & \bar{N}(r, 0 ; F)+\bar{N}(r, \infty ; F)+\bar{N}(r, 1 ; F)-\bar{N}\left(r, 0 ; F^{\prime}\right)+N_{k+2}\left(r, 0 ; F_{1}\right) \\
& -N_{2}(r, 0 ; F)-N\left(r, 0 ; L_{c}(f)\right)+S(r, f) \\
\leq \quad & N_{2}(r, 0 ; F)+N_{2}(r, 0 ; G)+\bar{N}(r, 0 ; F)+\frac{1}{2} \bar{N}(r, 0 ; G)+N_{k+2}\left(r, 0 ; F_{1}\right) \\
& -N_{2}(r, 0 ; F)-N\left(r, 0 ; L_{c}(f)\right)+S(r, f)+S(r, g) \\
\leq \quad & N_{k+2}\left(r, 0 ; F_{1}\right)+N_{k+2}\left(r, 0 ; G_{1}\right)+N_{k+1}\left(r, 0 ; F_{1}\right)+\frac{1}{2} N_{k+1}\left(r, 0 ; G_{1}\right) \\
& -N\left(r, 0 ; L_{c}(f)\right)+S(r, f)+S(r, g) \\
\leq \quad & (k+2)(\bar{N}(r, 0 ; f)+\bar{N}(r, 0 ; g))+2 N(r, 0 ; P(f))+N(r, 0 ; P(g)) \\
& +N\left(r, 0 ; L_{c}(g)\right)+(k+1) \bar{N}(r, 0 ; f)+N\left(r, 0 ; L_{c}(f)\right)+\frac{1}{2}(k+1) \bar{N}(r, 0 ; g) \\
& +\frac{1}{2} N(r, 0 ; P(g))+\frac{1}{2} N\left(r, 0 ; L_{c}(g)\right)+S(r, f)+S(r, g) \\
\leq \quad & (k+m+2)(T(r, f)+T(r, g))+(k+m+1) T(r, f)+T\left(r, L_{c}(f)\right) \\
& +T\left(r, L_{c}(g)\right)+\frac{1}{2}(k+m+1) T(r, g)+\frac{1}{2} T\left(r, L_{c}(g)\right)+S(r, f)+S(r, g) \\
\leq \quad & (k+m+2)(T(r, f)+T(r, g))+(k+m+1) T(r, f)+m\left(r, \frac{L_{c}(f)}{f}\right) \\
& +m(r, f)+m\left(r, \frac{L_{c}(g)}{g}\right)+m(r, g)+\frac{1}{2}(k+m+1) T(r, g)+\frac{1}{2} m\left(r, \frac{L_{c}(g)}{g}\right) \\
& +\frac{1}{2} m(r, g)+S(r, f)+S(r, g) \\
\leq \quad & (k+m+3)(T(r, f)+T(r, g))+(k+m+1) T(r, f)+\frac{1}{2}(k+m+2) T(r, g) \\
& +S(r, f)+S(r, g) .
\end{aligned}
$$


Similarly, we obtain

$$
\begin{aligned}
(n+m) T(r, g) \leq & (k+m+3)(T(r, f)+T(r, g))+(k+m+1) T(r, g) \\
& +\frac{1}{2}(k+m+2) T(r, f)+S(r, f)+S(r, g) .
\end{aligned}
$$

Combining (3.19) and (3.20), we get

$$
\left(n-\frac{7 k}{2}-\frac{5 m}{2}-8\right)(T(r, f)+T(r, g)) \leq S(r, f)+S(r, g),
$$

which is not possible since $n \geq \frac{7 k}{2}+\frac{5 m}{2}+9$.

Case 2: Let $H \equiv 0$. This case can be carried out similarly as done in case 2 of the proof of Theorem 1.1. So, we omit the details. This proves Theorem 1.3 .

\section{REFERENCES}

[1] A. Banerjee and S. Mukherjee, "Uniqueness of meromorphic functions concerning differential monomials sharing the same value", Bull. Math. Soc. Sci. Math. Roum. Nouv. Ser. 50, 191206 (2007).

[2] Y. M. Chiang and S. J. Feng, "On the Nevanlinna characteristic of $f(z+\eta)$ and difference equations in the complex plane" Ramanujan J. 16, 105-129 (2008).

[3] C. Y. Fang and M. L. Fang, "Uniqueness of meromorphic functions and differential polynomials", Comput. Math. Appl. 44, 607-617 (2002).

[4] W. K. Hayman, "Picard values of meromorphic Functions and their derivatives", Ann. Math. 70, 9-42 (1959).

[5] W. K. Hayman, "Meromorphic Functions" (The Clarendon Press, Oxford, 1964).

[6] I. Lahiri, "Value distribution of certain differential polynomials", Int. J. Math. Math. Sci. 28, 83-91 (2001).

[7] I. Lahiri, "Weighted value sharing and uniqueness of meromorphic functions", Complex Var. Theory Appl. 46, 241-253 (2001).

[8] I. Lahiri and A. Banerjee, "Weighted sharing of two sets", Kyungpook Math. J. 46 (1), 79-87 (2006).

[9] I. Lahiri and S. Dewan, "Value distribution of the product of a meromorphic function and its derivative", Kodai Math. J. 26, 95-100 (2003).

[10] I. Laine, "Nevanlinna Theory and Complex Differential Equations" (Walter de Gruyter, Berlin/Newyork, 1993).

[11] S. H. Lin and W. C. Lin, "Uniqueness of meromorphic functions concerning weakly weighted sharing", Kodai Math. J. 29, 269-280 (2006).

[12] X. Luo and W. C. Lin, "Value sharing results for shifts of meromorphic functions", J. Math. Anal. Appl. 377, 441-449 (2011).

[13] C. Meng, "Uniqueness of entire functions concerning difference polynomials", Math. Bohem. 139, 89-97 (2014).

[14] A. Z. Mohon'ko, "On the Nevanlinna characteristics of some meromorphic functions", Theory of Funct. Func. Anal. Appl. 14, 83-87 (1971).

[15] P. Sahoo, "Uniqueness of Entire Functions Related to Difference Polynomials", Commun. Math. Stat. 3, 227-238 (2015).

[16] P. Sahoo and G. Biswas, "Some results on uniqueness of entire functions concerning difference polynomials", Tamkang J. Math., 49 (2), 85-97 (2018).

[17] X. Y. Xu, K. Liu and T. B. Cao, "Uniqueness and value distribution for q-shifts of meromorphic functions", Math. Commun. 20, 97-112 (2015).

[18] L. Yang, "Value Distribution Theory" (Springer-Verlag and Science Press, 1993).

[19] C. C. Yang and X. H. Hua, "Uniqueness and value sharing of meromorphic functions", Ann. Acad. Sci. Fenn. Math. 22, 395-406 (1997).

[20] H. H. Yi and C. C. Yang, "Uniqueness Theory of Meromorphic Functions", Science Press, Beijing (1995).

[21] J. L. Zhang, "Value distribution and shared sets of differences of meromorphic functions", J. Math. Anal. Appl. 367, 401-408 (2010). 
[22] J. L. Zhang and L. Z. Yang, "Some results related to a conjecture of R. Bruck", J. Inequal. Pure Appl. Math. 8 (1) (2007), Art. 18, 11 pp.

Goutam Haldar

Department of Mathematics, Malda College, Rabindra Avenue, Malda, West Bengal 732101, INDIA.

Email address: goutamiit1986@gmail.com, goutamiitm@gmail.com 